ANNALES

POLONICI MATHEMATICI

99.1 (2010)

\title{
Stable harmonic maps between Finsler manifolds and Riemannian manifolds with positive Ricci curvature
}

\author{
by Jintang Li (Xiamen)
}

\begin{abstract}
We study the stability of harmonic maps between Finsler manifolds and Riemannian manifolds with positive Ricci curvature, and we prove that if $M^{n}$ is a compact Einstein Riemannian minimal submanifold of a Riemannian unit sphere with Ricci curvature satisfying $\operatorname{Ric}^{M}>n / 2$, then there is no non-degenerate stable harmonic map between $M$ and any compact Finsler manifold.
\end{abstract}

1. Introduction. Let $M$ be an $n$-dimensional smooth manifold and $\pi: T M \rightarrow M$ be the natural projection from the tangent bundle. Let $(x, Y)$ be a point of $T M$ with $x \in M, Y \in T_{x} M$ and let $\left(x^{i}, Y^{i}\right)$ be the local coordinates on $T M$ with $Y=Y^{i} \frac{\partial}{\partial x^{i}}$. A Finsler metric on $M$ is a function $F: T M \rightarrow[0,+\infty)$ with the following properties:

(i) regularity: $F(x, Y)$ is smooth in $T M \backslash 0$;

(ii) positive homogeneity: $F(x, \lambda Y)=\lambda F(x, Y)$ for $\lambda>0$;

(iii) strong convexity: the fundamental quadratic form $g=g_{i j} d x^{i} \otimes d x^{j}$ is positive-definite, where $g_{i j}=\frac{1}{2} \frac{\partial^{2}\left(F^{2}\right)}{\partial Y^{i} \partial Y^{j}}$.

Let $\phi: M \rightarrow \bar{M}$ be a non-degenerate (that is, $\operatorname{ker}(d \phi)=0$ ) smooth map between Finsler manifolds. Harmonic maps between Finsler manifolds are defined as the critical points of energy functionals. The first and second variation formulas for non-degenerate harmonic maps between Finsler manifolds were given in [HS] and [SZ]. As for stability of harmonic maps between Finsler manifolds, the results of He-Shen [HS] and Shen-Zhang [SZ] show that there is no non-degenerate stable harmonic map $M \rightarrow N$ if either the domain $M$ or the codomain $N$ is a Riemannian unit sphere $S^{n}(n>2)$. A direct generalization is to consider the non-existence of non-degenerate stable harmonic maps between arbitrary compact Finsler manifolds and

2010 Mathematics Subject Classification: 53C60, 58E20, 53B40.

Key words and phrases: harmonic maps, Finsler manifolds, stability. 
Riemannian manifolds with positive Ricci curvature. In this paper, we prove the following

TheOrem 1.1. Let $M^{n}(n \geq 3)$ be a compact Einstein Riemannian minimal submanifold of a Riemannian unit sphere. If the Ricci curvature satisfies $\mathrm{Ric}^{M}>n / 2$, then there is no non-degenerate stable harmonic map between $M$ and any compact Finsler manifold.

Remark. This theorem is obtained by He-Shen [HS] when $M$ is a Riemannian unit sphere.

COROLlary 1.2. There is no non-degenerate stable harmonic map between any compact Finsler manifold and a minimal Clifford hypersurface $S^{m}(\sqrt{1 / 2}) \times S^{m}(\sqrt{1 / 2})(m>2)$.

COROLlaRY 1.3. There is no non-degenerate stable harmonic map between any compact Finsler manifold and the Riemannian product $S^{m}(1 / 2) \times$ $S^{m}(1 / 2) \times S^{m}(1 / 2) \times S^{m}(1 / 2)(m>2)$.

2. Preliminaries. We shall use the following convention on index ranges unless otherwise stated:

$$
1 \leq i, j, \ldots \leq n ; \quad 1 \leq \alpha, \beta, \ldots \leq m ; \quad 1 \leq a, b, \ldots \leq n-1 .
$$

Let $(M, F)$ be an $n$-dimensional Finsler manifold. The Hilbert form and Cartan tensor are defined as follows:

$$
\omega^{n}=\frac{\partial F}{\partial Y^{i}} d x^{i}, \quad A=A_{i j k} d x^{i} \otimes d x^{j} \otimes d x^{k}, \quad A_{i j k}=F \frac{\partial g_{i j}}{\partial Y^{k}} .
$$

It is well known that there is a unique connection, the Chern connection, $\nabla$ on $\pi^{*} T M$ with $\nabla \frac{\partial}{\partial x^{i}}=\omega_{i}^{j} \frac{\partial}{\partial x^{j}}$ and $\omega_{i}^{j}=\Gamma_{i k}^{j} d x^{k}$ satisfying

$$
X\langle U, V\rangle=\left\langle\nabla_{X} U, V\right\rangle+\left\langle U, \nabla_{X} V\right\rangle+2 C\left(U, V, \nabla_{X}\left(F e_{n}\right)\right),
$$

where $A_{i j k}=F C_{i j k}$ and $X, U, V \in \Gamma\left(\pi^{*} T M\right)$.

The curvature 2 -forms of the Chern connection $\nabla$ are

$$
\omega_{j}^{i}-\omega_{j}^{k} \wedge \omega_{k}^{i}=\Omega_{j}^{i}=\frac{1}{2} R_{j k l}^{i} d x^{k} \wedge d x^{l}+\frac{1}{F} P_{j k l}^{i} d x^{k} \wedge \delta Y^{l},
$$

where $\delta Y^{i}=d Y^{i}+N_{j}^{i} d x^{j}, N_{j}^{i}=\gamma_{j k}^{i} Y^{k}-\frac{1}{F} A_{j k}^{i} \gamma_{s t}^{k} Y^{s} Y^{t}$ and $\gamma_{j k}^{i}$ are the formal Christoffel symbols of the second kind for $g_{i j}$.

Take a $g$-orthonormal frame $\left\{e_{i}=u_{i}^{j} \frac{\partial}{\partial x^{j}}\right\}$ with $e_{n}=\hat{e}=Y / F$ for each fibre of $\pi^{*} T M$ and let $\left\{\omega^{i}\right\}$ be its dual coframe. The collection $\left\{\omega^{i}, \omega_{n}^{i}\right\}$ forms an orthonormal basis for $T^{*}(T M \backslash\{0\})$ with respect to the Sasaki type metric $g_{i j} d x^{i} \otimes d x^{j}+g_{i j} \delta Y^{i} \otimes \delta Y^{j}$. The pull-back of the Sasaki metric from $T M \backslash\{0\}$ to $S M$ is a Riemannian metric

$$
\widehat{g}=g_{i j} d x^{i} \otimes d x^{j}+\delta_{a b} \omega_{n}^{a} \otimes \omega_{n}^{b} .
$$

Then we have 
Lemma 2.1 ([M, Lemma 2.2]). For $\psi=\psi_{i} \omega^{i} \in \Gamma\left(\pi^{*} T^{*} M\right)$, we have

$$
\operatorname{div}_{\widehat{g}} \psi=\sum_{i} \psi_{i \mid i}+\sum_{a, b} \psi_{a} P_{b b a},
$$

where | denotes the horizontal covariant differential with respect to the Chern connection, $e_{i}^{H}=u_{i}^{j} \frac{\delta}{\delta x^{j}}=u_{i}^{j}\left(\frac{\partial}{\partial x^{j}}-N_{j}^{k} \frac{\partial}{\partial Y^{k}}\right)$ denotes the horizontal part of $e_{i}$ and $P_{b b a}=P_{b b a}^{n}$.

Let $\phi: M^{n} \rightarrow \bar{M}^{m}$ be a non-degenerate smooth map between Finsler manifolds. The energy density of $\phi$ is the function $e(\phi): S M \rightarrow \mathbb{R}$ defined by

$$
e(\phi)(x, Y)=\frac{1}{2} g^{i j}(x, Y) \phi_{i}^{\alpha} \phi_{j}^{\beta} \bar{g}_{\alpha \beta}(\bar{x}, \bar{Y}),
$$

where $d \phi\left(\frac{\partial}{\partial x^{i}}\right)=\phi_{i}^{\alpha} \frac{\partial}{\partial \bar{x}^{\alpha}}$ and $\bar{Y}=\bar{Y}^{\alpha} \frac{\partial}{\partial \bar{x}^{\alpha}}=Y^{i} \phi_{i}^{\alpha} \frac{\partial}{\partial \bar{x}^{\alpha}}$.

We define the energy functional $E(\phi)$ by

$$
E(\phi)=\frac{1}{C_{n-1}} \int_{S M} e(\phi) d V_{S M},
$$

where $d V_{S M}=\Omega d \tau \wedge d x, \Omega=\operatorname{det}\left(g_{i j} / F\right), d \tau=\sum_{i}(-1)^{i-1} Y^{i} d Y^{1} \wedge \cdots \wedge$ $\widehat{d Y^{i}} \wedge \cdots \wedge d Y^{n}, d x=d x^{1} \wedge \cdots \wedge d x^{n}$ and $C_{n-1}$ denotes the volume of the unit Euclidean sphere $S^{n-1}$.

We call $\phi$ a harmonic map if it is a critical point of the energy functional. Let $\widetilde{\nabla}$ be the pullback Chern connection on $\pi^{*}\left(\phi^{-1} T \bar{M}\right)$. We introduce the tension field $\tau$ of $\phi$ and a section $J(\bar{U}, d \phi, \bar{V}) \in C\left(T^{*} M \otimes \phi^{-1} T \bar{M}\right)$ as follows:

$$
\begin{aligned}
\tau= & \sum_{i}\left(\widetilde{\nabla}_{e_{i}^{H}} d \phi\right) e_{i}+\sum_{i, \alpha}\left\{2 \bar{C}\left(\bar{e}_{\alpha}, d \phi e_{i}, \widetilde{\nabla}_{e_{i}^{H}}\left(d \phi F e_{n}\right)\right) \bar{e}_{\alpha}\right. \\
& \left.+\left(\widetilde{\nabla}_{F e_{n}^{H}} \bar{C}\right)\left(d \phi e_{i}, d \phi e_{i}, \bar{e}_{\alpha}\right) \bar{e}_{\alpha}+2 \bar{C}\left(\widetilde{\nabla}_{F e_{n}^{H}}\left(d \phi e_{i}\right), d \phi e_{i}, \bar{e}_{\alpha}\right) \bar{e}_{\alpha}\right\} \\
& +\sum_{a, b}\left\langle\bar{e}_{\alpha}, d \phi e_{b}\right\rangle \bar{e}_{\alpha} P_{a a b}
\end{aligned}
$$

and

$$
J(\bar{U}, d \phi, \bar{V})=\bar{R}(\bar{U}, d \phi) \bar{V}+\frac{F}{\bar{F}} \bar{P}\left(\bar{U}, \widetilde{\nabla}_{\hat{e}} d \phi\right) \bar{V}-\frac{F}{\bar{F}} \bar{P}\left(d \phi, \widetilde{\nabla}_{\hat{e}} \bar{U}\right) \bar{V} .
$$

We have

Theorem 2.2 ([SZ, Theorem 2.1]). A map $\phi$ is harmonic if and only if

$$
\int_{S M}\langle V, \tau\rangle d V_{S M}=0
$$

for any vector $V \in \Gamma\left(\phi^{-1} T \bar{M}\right)$.

Theorem 2.3 ([SZ, Theorem 3.1]). Let $\phi: M^{n} \rightarrow \bar{M}^{m}$ be a nondegenerate harmonic map. Let $\phi_{t}$ be a smooth variation of $\phi$ with $\phi_{0}=\phi$ 
and $V=\left.\frac{\partial \phi_{t}}{\partial t}\right|_{t=0}$. Then the second variation of the energy functional for $\phi$ is

$$
I(V, V)=\left.\frac{d^{2}}{d t^{2}} E\left(\phi_{t}\right)\right|_{t=0}=\Xi_{1}+\Xi_{2}+\Xi_{3}+\Xi_{4}+\Xi_{5},
$$

where

$$
\begin{aligned}
\Xi_{1}= & \frac{1}{C_{n-1}} \int_{S M}\|\widetilde{\nabla} V\|^{2} d V_{S M}, \\
\Xi_{2}= & \frac{1}{C_{n-1}} \int_{S M} \operatorname{Tr}_{g}\langle J(V, d \phi, V), d \phi\rangle d V_{S M}, \\
\Xi_{3}= & \frac{1}{C_{n-1}} \int_{S M} \operatorname{Tr}_{g} \bar{C}\left(d \phi, d \phi, J\left(V, d \phi\left(F e_{n}\right), V\right)\right) d V_{S M}, \\
\Xi_{4}= & -\frac{1}{C_{n-1}} \int_{S M}\left\langle\widetilde{\nabla}_{V} V, \tau\right\rangle d V_{S M}, \\
\Xi_{5}= & \frac{1}{C_{n-1}} \int_{S M}\left\{\operatorname{Tr}_{g}\left(\widetilde{\nabla}_{V H} \bar{C}\right)\left(d \phi, d \phi, \widetilde{\nabla}_{F \hat{e}} V\right)\right. \\
& +\operatorname{Tr}_{g} \overline{\mathcal{C}}\left(d \phi, d \phi, \widetilde{\nabla}_{F \hat{e}} V, \widetilde{\nabla}_{F \hat{e}} V\right) \\
& \left.+4 \operatorname{Tr}_{g} \bar{C}\left(\widetilde{\nabla} V, d \phi, \widetilde{\nabla}_{F \hat{e}} V\right)\right\} d V_{S M} .
\end{aligned}
$$

3. The stability. Let $x: M^{n} \rightarrow S^{n+p} \rightarrow E^{n+p+1}$ be a compact Riemannian minimal submanifold of the Riemannian unit sphere $S^{n+p}$. We choose a local field of orthonormal frames $\left\{e_{0}, \ldots, e_{n+p}\right\}$ in the Euclidean space $E^{n+p+1}$ such that $\left\{e_{1}, \ldots, e_{n}\right\}$ are tangent to $M$ and $x=e_{0}$. Then we have

$$
\begin{aligned}
d e_{i} & =\omega_{i}^{j} e_{j}+h_{i j}^{\alpha} e_{\alpha} \omega^{j}-\omega^{i} x, \\
d e_{\mu} & =-h_{i j}^{\mu} \omega^{j} e_{i}+\omega_{\mu}^{\nu} e_{\nu} \quad(\mu, \nu=n+1, \ldots, n+p),
\end{aligned}
$$

where $B=h_{i j}^{\mu} \omega^{i} \otimes \omega^{j} \otimes e_{\mu}$ is the second fundamental form of $M$ in $S^{n+P}$. Let $\left\{\Lambda_{1}, \ldots, \Lambda_{n+p+1}\right\}$ be the constant orthonormal basis in $E^{n+p+1}$ and let $V_{A}=\left\langle\Lambda_{A}, e_{i}\right\rangle e_{i}, A=1, \ldots, n+p+1$. A straightforward computation shows

$$
\nabla_{e_{i}} V_{A}=\sum_{\mu, j} v_{A}^{\mu} h_{i j}^{\mu} e_{j}-\left\langle\Lambda_{A}, e_{0}\right\rangle e_{i}
$$

where $v_{A}^{\mu}=\left\langle\Lambda_{A}, e_{\mu}\right\rangle$.

First, we assume that the source manifold is $M$. The second variation formula for the harmonic map $\phi: M \rightarrow \bar{M}^{m}$ can be written as

$$
\sum_{A} I\left(d \phi V_{A}, d \phi V_{A}\right)=\Xi_{1}+\Xi_{2}+\Xi_{3}+\Xi_{4}+\Xi_{5}
$$


where

$$
\begin{aligned}
\Xi_{1}= & \sum_{A} \frac{1}{C_{n-1}} \int_{S M}\left\|\widetilde{\nabla}\left(d \phi V_{A}\right)\right\|^{2} d V_{S M}, \\
\Xi_{2}= & \sum_{A} \frac{1}{C_{n-1}} \int_{S M} \operatorname{Tr}_{g}\langle J(d \phi V, d \phi, d \phi V), d \phi\rangle d V_{S M}, \\
\Xi_{3}= & \sum_{A} \frac{1}{C_{n-1}} \int_{S M} \operatorname{Tr}_{g} \bar{C}\left(d \phi, d \phi, J\left(d \phi V, d \phi\left(F e_{n}\right), d \phi V\right)\right) d V_{S M}, \\
\Xi_{4}= & -\sum_{A} \frac{1}{C_{n-1}} \int_{S M}\left\langle\widetilde{\nabla}_{d \phi V_{A}}\left(d \phi V_{A}\right), \tau\right\rangle d V_{S M} \\
\Xi_{5}= & \sum_{A} \frac{1}{C_{n-1}} \int_{S M}\left\{\operatorname{Tr}_{g}\left(\widetilde{\nabla}_{\left(d \phi V_{A}\right)^{H}} \bar{C}\right)\left(d \phi, d \phi, \widetilde{\nabla}_{V_{A}}(d \phi F \hat{e})\right)\right. \\
& +\operatorname{Tr}_{g} \overline{\mathcal{C}}\left(d \phi, d \phi, \widetilde{\nabla}_{V_{A}}(d \phi F \hat{e}), \widetilde{\nabla}_{V_{A}}(d \phi F \hat{e})\right) \\
& \left.+4 \operatorname{Tr}_{g} \bar{C}\left(\widetilde{\nabla}\left(d \phi V_{A}\right), d \phi, \widetilde{\nabla}_{V_{A}}(d \phi F \hat{e})\right)\right\} d V_{S M} .
\end{aligned}
$$

We have [HS, (3.4)]

$$
\begin{aligned}
\left(\widetilde{\nabla}_{X^{H}} \widetilde{\nabla}_{Z} d \phi\right) Y= & -d \phi R(X, Y) Z+\left(\widetilde{\nabla}_{Y^{H}} \widetilde{\nabla}_{Z} d \phi\right) X \\
& +\left(\widetilde{\nabla}_{Y} d \phi\right)\left(\nabla_{X^{H}} Z\right)-\left(\widetilde{\nabla}_{X} d \phi\right)\left(\nabla_{Y^{H}} Z\right) \\
& +\bar{R}(d \phi X, d \phi Y) d \phi Z+\frac{F}{\bar{F}} \bar{P}\left(d \phi X,\left(\widetilde{\nabla}_{e_{n}} d \phi\right) Y\right) d \phi Z \\
& -\frac{F}{\bar{F}} \bar{P}\left(d \phi Y,\left(\widetilde{\nabla}_{e_{n}} d \phi\right) X\right) d \phi Z
\end{aligned}
$$

Set $X=Z=V, Y=e_{i}$ in (3.7). We obtain

$$
\begin{aligned}
-\left\langle\bar{R}\left(d \phi e_{i}, d \phi V\right) d \phi V, d \phi e_{i}\right\rangle+\frac{F}{\bar{F}}\left\langle\bar{P}\left(d \phi V,\left(\widetilde{\nabla}_{e_{i}} d \phi\right) e_{n}\right) d \phi V, d \phi e_{i}\right\rangle \\
-\frac{F}{\bar{F}}\left\langle\bar{P}\left(d \phi e_{i},\left(\widetilde{\nabla}_{e_{n}} d \phi\right) V\right) d \phi V, d \phi e_{i}\right\rangle \\
=-\left\langle d \phi R\left(e_{i}, V\right) V, d \phi e_{i}\right\rangle+\left\langle\left(\widetilde{\nabla}_{V^{H}} \widetilde{\nabla}_{V} d \phi\right) e_{i}, d \phi e_{i}\right\rangle \\
-\left\langle\left(\widetilde{\nabla}_{e_{i}^{H}} \widetilde{\nabla}_{V} d \phi\right) V, d \phi e_{i}\right\rangle-\left\langle\left(\widetilde{\nabla}_{e_{i}} d \phi\right)\left(\nabla_{V^{H}} V\right), d \phi e_{i}\right\rangle \\
+\left\langle\left(\widetilde{\nabla}_{V} d \phi\right)\left(\nabla_{e_{i}^{H}} V\right), d \phi e_{i}\right\rangle .
\end{aligned}
$$

Substituting (3.8) into (3.3) yields

$$
\begin{aligned}
\Xi_{2}= & \sum_{A} \frac{1}{C_{n-1}} \int_{S M}\left\{-\left\langle d \phi R\left(e_{i}, V_{A}\right) V_{A}, d \phi e_{i}\right\rangle\right. \\
& +\left\langle\left(\widetilde{\nabla}_{V_{A}^{H}} \widetilde{\nabla}_{V_{A}} d \phi\right) e_{i}, d \phi e_{i}\right\rangle \\
& -\left\langle\left(\widetilde{\nabla}_{e_{i}^{H}} \widetilde{\nabla}_{V_{A}} d \phi\right) V_{A}, d \phi e_{i}\right\rangle-\left\langle\left(\widetilde{\nabla}_{e_{i}} d \phi\right)\left(\nabla_{V_{A}^{H}} V_{A}\right), d \phi e_{i}\right\rangle \\
& \left.+\left\langle\left(\widetilde{\nabla}_{V_{A}} d \phi\right)\left(\nabla_{e_{i}^{H}} V_{A}\right), d \phi e_{i}\right\rangle\right\} d V_{S M} .
\end{aligned}
$$


Similarly, set $X=Z=V_{A}, Y=F e_{n}$ in (3.7). We also obtain

$$
\begin{aligned}
-\bar{R}\left(d \phi F e_{n}, d \phi V_{A}\right) d & \phi V_{A}+\frac{F}{\bar{F}} \bar{P}\left(d \phi V_{A},\left(\widetilde{\nabla}_{F e_{n}} d \phi\right) e_{n}\right) d \phi V_{A} \\
& -\frac{F}{\bar{F}} \bar{P}\left(d \phi F e_{n},\left(\widetilde{\nabla}_{e_{n}} d \phi\right) V_{A}\right) d \phi V_{A} \\
= & -d \phi R\left(F e_{n}, V_{A}\right) V_{A}+\left(\widetilde{\nabla}_{V_{A}^{H}} \widetilde{\nabla}_{V_{A}} d \phi\right) F e_{n} \\
& -\left(\widetilde{\nabla}_{F e_{n}^{H}} \widetilde{\nabla}_{V_{A}} d \phi\right) V_{A}-\left(\widetilde{\nabla}_{F e_{n}} d \phi\right)\left(\nabla_{V_{A}^{H}} V_{A}\right) \\
& +\left(\widetilde{\nabla}_{V_{A}} d \phi\right)\left(\nabla_{F e_{n}^{H}} V_{A}\right) .
\end{aligned}
$$

Substituting (3.10) into (3.4) yields

$$
\begin{aligned}
\Xi_{3}= & \sum_{A} \frac{1}{C_{n-1}} \int_{S M}\left\{-\bar{C}\left(d \phi e_{i}, d \phi e_{i}, d \phi R\left(F e_{n}, V_{A}\right) V_{A}\right)\right. \\
& +\bar{C}\left(d \phi e_{i}, d \phi e_{i},\left(\widetilde{\nabla}_{V_{A}^{H}} \widetilde{\nabla}_{V_{A}} d \phi\right) F e_{n}\right) \\
& -\bar{C}\left(d \phi e_{i}, d \phi e_{i},\left(\widetilde{\nabla}_{F e_{n}^{H}} \widetilde{\nabla}_{V_{A}} d \phi\right) V_{A}\right) \\
& -\bar{C}\left(d \phi e_{i}, d \phi e_{i}+\left(\widetilde{\nabla}_{F e_{n}} d \phi\right)\left(\nabla_{V_{A}^{H}} V_{A}\right)\right) \\
& \left.+\bar{C}\left(d \phi e_{i}, d \phi e_{i},\left(\widetilde{\nabla}_{V_{A}} d \phi\right)\left(\nabla_{F e_{n}^{H}} V_{A}\right)\right)\right\} d V_{S M}
\end{aligned}
$$

A direct calculation gives

LEMMA 3.1.

$$
\begin{aligned}
\Xi_{2}= & \sum_{A} \frac{1}{C_{n-1}} \int_{S M}\left\{-\left\langle d \phi R\left(e_{i}, V_{A}\right) V_{A}, d \phi e_{i}\right\rangle-\left\langle\widetilde{\nabla}_{e_{i}^{H}}\left\{\left(\widetilde{\nabla}_{V_{A}} d \phi\right) V_{A}\right\}, d \phi e_{i}\right\rangle\right. \\
& \left.+\left\langle\widetilde{\nabla}_{V_{A}^{H}}\left[\left(\widetilde{\nabla}_{V_{A}} d \phi\right) e_{i}\right], d \phi e_{i}\right\rangle-\left\langle\left(\widetilde{\nabla}_{V_{A}} d \phi\right)\left(\nabla_{V_{A}^{H}} e_{i}\right), d \phi e_{i}\right\rangle\right\} d V_{S M} .
\end{aligned}
$$

LEMMA 3.2 .

$$
\begin{aligned}
\Xi_{3}= & \sum_{A} \frac{1}{C_{n-1}} \int_{S M}\left\{-\bar{C}\left(d \phi e_{i}, d \phi e_{i}, d \phi R\left(F e_{n}, V_{A}\right) V_{A}\right)\right. \\
& -\bar{C}\left(d \phi e_{i}, d \phi e_{i}, \widetilde{\nabla}_{F e_{n}^{H}}\left\{\left(\widetilde{\nabla}_{V_{A}} d \phi\right) V_{A}\right\}\right) \\
& \left.+\bar{C}\left(d \phi e_{i}, d \phi e_{i}, \widetilde{\nabla}_{V_{A}^{H}}\left[\left(\widetilde{\nabla}_{V_{A}} d \phi\right) F e_{n}\right)\right]\right\} d V_{S M} .
\end{aligned}
$$

We need the following

LEMMA 3.3.

$$
\begin{aligned}
-\sum_{A} \int_{S M}\left\{\left\langle\widetilde{\nabla}_{e_{i}^{H}}\left[\left(\widetilde{\nabla}_{V_{A}} d \phi\right) V_{A}\right], d \phi e_{i}\right\rangle\right. \\
\left.+\bar{C}\left(d \phi e_{i}, d \phi e_{i}, \widetilde{\nabla}_{F e_{n}^{H}}\left[\left(\widetilde{\nabla}_{V_{A}} d \phi\right) V_{A}\right]\right)\right\} d V_{S M}+\Xi_{4}=0 .
\end{aligned}
$$


Proof. Consider $\psi=\bar{C}\left(d \phi e_{i}, d \phi e_{i},\left(\widetilde{\nabla}_{V_{A}} d \phi\right) V_{A}\right) F \omega^{n}$. We get

$$
\begin{aligned}
\operatorname{div}_{\widehat{g}} \psi= & \left(\widetilde{\nabla}_{F e_{n}^{H}} \bar{C}\right)\left(d \phi e_{i}, d \phi e_{i},\left(\widetilde{\nabla}_{V_{A}} d \phi\right) V_{A}\right) \\
& +2 \bar{C}\left(\widetilde{\nabla}_{F e_{n}^{H}} d \phi e_{i}, d \phi e_{i},\left(\widetilde{\nabla}_{V_{A}} d \phi\right) V_{A}\right) \\
& +\bar{C}\left(d \phi e_{i}, d \phi e_{i}, \widetilde{\nabla}_{F e_{n}^{H}}\left[\left(\widetilde{\nabla}_{V_{A}} d \phi\right) V_{A}\right]\right) .
\end{aligned}
$$

Integrating (3.12) yields

$$
\begin{aligned}
\sum_{A} \int_{S M} \bar{C}\left(d \phi e_{i}, d \phi e_{i}, \widetilde{\nabla}_{F e_{n}^{H}}\left[\left(\widetilde{\nabla}_{V_{A}} d \phi\right) V_{A}\right]\right) d V_{S M} \\
=-\sum_{A} \int_{S M}\left\{\left(\widetilde{\nabla}_{F e_{n}^{H}} \bar{C}\right)\left(d \phi e_{i}, d \phi e_{i},\left(\widetilde{\nabla}_{V_{A}} d \phi\right) V_{A}\right)\right. \\
\left.+2 \bar{C}\left(\widetilde{\nabla}_{F e_{n}^{H}} d \phi e_{i}, d \phi e_{i},\left(\widetilde{\nabla}_{V_{A}} d \phi\right) V_{A}\right)\right\} d V_{S M} .
\end{aligned}
$$

Similarly, we also have

$$
\begin{aligned}
\sum_{A} \int_{S M}\left\langle\widetilde{\nabla}_{e_{i}^{H}}\left[\left(\widetilde{\nabla}_{V_{A}} d \phi\right) V_{A}\right], d \phi e_{i}\right\rangle d V_{S M} \\
=-\sum_{A} \int_{S M}\left\{\left\langle\left(\widetilde{\nabla}_{V_{A}} d \phi\right) V_{A},\left(\widetilde{\nabla}_{e_{i}^{H}} d \phi\right) e_{i}\right\rangle\right. \\
\left.+2 \bar{C}\left(\left(\widetilde{\nabla}_{V_{A}} d \phi\right) V_{A}, d \phi e_{i}, \widetilde{\nabla}_{e_{i}^{H}} d \phi F e_{n}\right)\right\} d V_{S M} .
\end{aligned}
$$

Because $\phi$ is a harmonic map, by (3.13) and (3.14), we immediately obtain

$$
\begin{aligned}
-\sum_{A} \int_{S M}\left\{\bar{C}\left(d \phi e_{i}, d \phi e_{i}, \nabla_{F e_{n}^{H}}\left[\left(\widetilde{\nabla}_{V_{A}} d \phi\right) V_{A}\right)\right]\right. \\
\left.+\left\langle\widetilde{\nabla}_{e_{i}^{H}}\left[\left(\widetilde{\nabla}_{V_{A}} d \phi\right) V_{A}\right], d \phi e_{i}\right\rangle\right\} d V_{S M}+\Xi_{4}=0,
\end{aligned}
$$

which completes the proof of Lemma 3.3.

If $M$ is Einstein, then we have

$$
\bar{C}\left(d \phi e_{i}, d \phi e_{i}, d \phi R\left(F e_{n}, V_{A}\right) V_{A}\right)=0 .
$$

It follows from (3.16) and Lemmas 3.1-3.3 that

$$
\begin{aligned}
\Xi_{2}+\Xi_{3}+\Xi_{4}= & \sum_{A} \frac{1}{C_{n-1}} \int_{S M}\left\{-\left\langle d \phi R\left(e_{i}, V_{A}\right) V_{A}, d \phi e_{i}\right\rangle\right. \\
& +\left\langle\widetilde{\nabla}_{V_{A}^{H}}\left[\left(\widetilde{\nabla}_{V_{A}} d \phi\right) e_{i}\right], d \phi e_{i}\right\rangle-\left\langle\left(\widetilde{\nabla}_{V_{A}} d \phi\right)\left(\nabla_{V_{A}^{H}} e_{i}\right), d \phi e_{i}\right\rangle \\
& \left.+\bar{C}\left(d \phi e_{i}, d \phi e_{i}, \widetilde{\nabla}_{V_{A}^{H}}\left[\left(\widetilde{\nabla}_{V_{A}} d \phi\right) F e_{n}\right]\right)\right\} d V_{S M} .
\end{aligned}
$$


LEMMA 3.4.

$$
\begin{aligned}
\sum_{A} \frac{1}{C_{n-1}} \int_{S M}\left\langle\widetilde{\nabla}_{V_{A}^{H}}\left\{\left(\widetilde{\nabla}_{V_{A}} d \phi\right) e_{i}\right\}, d \phi e_{i}\right\rangle d V_{S M}+\Xi_{1} \\
=\frac{1}{C_{n-1}} \int_{S M}\left\{h_{i j}^{\mu} h_{i k}^{\mu}\left\langle d \phi e_{j}, d \phi e_{k}\right\rangle-\sum_{A}\left\langle d \phi\left(\nabla_{V_{A}^{H}} e_{i}\right),\left(\widetilde{\nabla}_{V_{A}} d \phi\right) e_{i}\right)\right\rangle \\
\left.\quad-\sum_{A} 2 \bar{C}\left(\left(\widetilde{\nabla}_{V_{A}} d \phi\right) e_{i}, d \phi e_{i}, \widetilde{\nabla}_{V_{A}^{H}}\left(d \phi F e_{n}\right)\right)+|d \phi|^{2}\right\} d V_{S M} .
\end{aligned}
$$

Proof. Let $\psi=\sum_{A, i, j}\left\langle\left(\widetilde{\nabla}_{V_{A}} d \phi\right) e_{i}, d \phi e_{i}\right\rangle v_{A}^{j} \omega^{j}$. We have

$$
\begin{array}{r}
\operatorname{div}_{\widehat{g}} \psi=\sum_{A}\left\{\left\langle\nabla_{V_{A}^{H}}\left\{\left(\widetilde{\nabla}_{V_{A}} d \phi\right) e_{i}\right\}, d \phi e_{i}\right\rangle+\left\langle\left(\widetilde{\nabla}_{V_{A}} d \phi\right) e_{i}, \nabla_{V_{A}^{H}}\left(d \phi e_{i}\right)\right\rangle\right. \\
\left.+2 \bar{C}\left(\left(\widetilde{\nabla}_{V_{A}} d \phi\right) e_{i}, d \phi e_{i}, \widetilde{\nabla}_{V_{A}^{H}}\left(d \phi F e_{n}\right)\right)\right\} .
\end{array}
$$

Integrating (3.18) implies

$$
\begin{aligned}
\sum_{A} \int_{S M}\left\langle\widetilde{\nabla}_{V_{A}^{H}}\left\{\left(\widetilde{\nabla}_{V_{A}} d \phi\right) e_{i}\right\}, d \phi e_{i}\right\rangle d V_{S M} \\
=-\sum_{A} \int_{S M}\left\{\left\langle\left(\widetilde{\nabla}_{V_{A}} d \phi\right) e_{i}, \nabla_{V_{A}^{H}}\left(d \phi e_{i}\right)\right\rangle\right. \\
\left.\quad+2 \bar{C}\left(\widetilde{\nabla}_{e_{i}}\left(d \phi V_{A}\right), d \phi e_{i}, \widetilde{\nabla}_{V_{A}^{H}}\left(d \phi F e_{n}\right)\right)\right\} d V_{S M} .
\end{aligned}
$$

On the other hand, we also have

$$
\begin{aligned}
\Xi_{1}= & \frac{1}{C_{n-1}} \int_{S M}\left\{\sum_{A}\left\langle\widetilde{\nabla}_{V_{A}^{H}}\left(d \phi e_{i}\right),\left(\widetilde{\nabla}_{V_{A}} d \phi\right) e_{i}\right)\right\rangle \\
& \left.-\sum_{A}\left\langle d \phi\left(\nabla_{V_{A}^{H}} e_{i}\right),\left(\widetilde{\nabla}_{V_{A}} d \phi\right) e_{i}\right)\right\rangle \\
& \left.+h_{i j}^{\mu} h_{i k}^{\mu}\left\langle d \phi e_{j}, d \phi e_{k}\right\rangle+|d \phi|^{2}\right\} d V_{S M} .
\end{aligned}
$$

Combining (3.19) and (3.20) shows Lemma 3.4.

Substituting the formula of Lemma 3.4 into (3.17), we get

$$
\begin{aligned}
\Xi_{1} & +\Xi_{2}+\Xi_{3}+\Xi_{4} \\
= & \frac{1}{C_{n-1}} \int_{S M}\left\{-\sum_{A}\left\langle d \phi R\left(e_{i}, V_{A}\right) V_{A}, d \phi e_{i}\right\rangle+h_{i j}^{\mu} h_{i k}^{\mu}\left\langle d \phi e_{j}, d \phi e_{k}\right\rangle\right. \\
& +\sum_{A} \bar{C}\left(d \phi e_{i}, d \phi e_{i}, \widetilde{\nabla}_{V_{A}^{H}}\left[\left(\widetilde{\nabla}_{V_{A}} d \phi\right) F e_{n}\right]\right)+|d \phi|^{2} \\
& \left.-2 \bar{C}\left(\widetilde{\nabla}_{e_{i}}\left(d \phi V_{A}\right), d \phi e_{i}, \widetilde{\nabla}_{V_{A}^{H}}\left(d \phi F e_{n}\right)\right)\right\} d V_{S M} .
\end{aligned}
$$


Obviously, we can obtain

$$
\begin{aligned}
\sum_{A} \frac{1}{C_{n-1}} \int_{S M} \bar{C}\left(d \phi e_{i}, d \phi e_{i}, \widetilde{\nabla}_{V_{A}^{H}}\left[\left(\widetilde{\nabla}_{V_{A}} d \phi\right) F e_{n}\right]\right) d V_{S M} \\
=-\sum_{A} \frac{1}{C_{n-1}} \int_{S M}\left\{\left(\widetilde{\nabla}_{V_{A}^{H}} \bar{C}\right)\left(d \phi e_{i}, d \phi e_{i}, \widetilde{\nabla}_{F e_{n}}\left(d \phi V_{A}\right)\right)\right. \\
\left.\quad+2 \bar{C}\left(\widetilde{\nabla}_{V_{A}} d \phi e_{i}, d \phi e_{i},\left(\widetilde{\nabla}_{V_{A}} d \phi\right) F e_{n}\right)\right\} d V_{S M} .
\end{aligned}
$$

Substituting (3.22) into (3.21), we get

$$
\begin{aligned}
\Xi_{1} & +\Xi_{2}+\Xi_{3}+\Xi_{4} \\
= & \frac{1}{C_{n-1}} \int_{S M}\left\{-\sum_{A}\left\langle d \phi R\left(e_{i}, V_{A}\right) V_{A}, d \phi e_{i}\right\rangle+h_{i j}^{\mu} h_{i k}^{\mu}\left\langle d \phi e_{j}, d \phi e_{k}\right\rangle\right. \\
& -\sum_{A}\left(\widetilde{\nabla}_{V_{A}^{H}} \bar{C}\right)\left(d \phi e_{i}, d \phi e_{i}, \widetilde{\nabla}_{F e_{n}}\left(d \phi V_{A}\right)\right) \\
& \left.-4 \sum_{A} \bar{C}\left(\widetilde{\nabla}_{e_{i}^{H}}\left(d \phi V_{A}\right), d \phi e_{i}, \widetilde{\nabla}_{V_{A}}\left(d \phi F e_{n}\right)\right)+|d \phi|^{2}\right\} d V_{S M} .
\end{aligned}
$$

On the other hand, we have

$$
\begin{aligned}
\sum_{A} & \frac{1}{C_{n-1}} \int_{S M}\left\{\left(\widetilde{\nabla}_{\left.\left(d \phi V_{A}\right)^{H} \bar{C}\right)\left(d \phi e_{i}, d \phi e_{i}, \widetilde{\nabla}_{F e_{n}} d \phi V_{A}\right)}\right.\right. \\
& \left.+\overline{\mathcal{C}}\left(d \phi e_{i}, d \phi e_{i}, \widetilde{\nabla}_{F e_{n}} d \phi V_{A}, \widetilde{\nabla}_{F e_{n}} d \phi V_{A}\right)\right\} d V_{S M} \\
= & \sum_{A} \frac{1}{C_{n-1}} \int_{S M}\left\{\left(\widetilde{\nabla}_{v_{A}^{k} u_{k}^{l} \phi_{l}^{\alpha}\left(\frac{\partial}{\partial \bar{x}^{\alpha}}-\bar{N}_{\alpha}^{\beta} \frac{\partial}{\partial \bar{Y}^{\beta}}\right)} \bar{C}\right)\left(d \phi e_{i}, d \phi e_{i}, \widetilde{\nabla}_{F e_{n}} d \phi V_{A}\right)\right. \\
& \left.+\overline{\mathcal{C}}\left(d \phi e_{i}, d \phi e_{i}, \widetilde{\nabla}_{F e_{n}} d \phi V_{A}, \widetilde{\nabla}_{v_{A}^{k} u_{k}^{l}\left(\frac{\partial}{\partial x^{l}}-N_{l}^{j} \frac{\partial}{\partial Y^{j}}\right)} d \phi F e_{n}\right)\right\} d V_{S M} \\
= & \sum_{A} \frac{1}{C_{n-1}} \int_{S M}\left(\widetilde{\nabla}_{V_{A}^{H}} \bar{C}\right)\left(d \phi e_{i}, d \phi e_{i}, \widetilde{\nabla}_{F e_{n}}\left(d \phi V_{A}\right)\right) d V_{S M} .
\end{aligned}
$$

It follows from (3.24) and (3.7) that

$$
\begin{aligned}
\Xi_{5}= & \sum_{A} \frac{1}{C_{n-1}} \int_{S M}\left\{\left(\widetilde{\nabla}_{V_{A}^{H}} \bar{C}\right)\left(d \phi e_{i}, d \phi e_{i}, \widetilde{\nabla}_{F e_{n}}\left(d \phi V_{A}\right)\right)\right. \\
& \left.+4 \bar{C}\left(\widetilde{\nabla}_{e_{i}^{H}}\left(d \phi V_{A}\right), d \phi e_{i},\left(\widetilde{\nabla}_{V_{A}} d \phi\right) F e_{n}\right)\right\} d V_{S M} .
\end{aligned}
$$

Combining (3.23) and (3.25) yields

$$
\sum_{A} I\left(d \phi V_{A}, d \phi V_{A}\right)=\frac{1}{C_{n-1}} \int_{S M}\left\{n|d \phi|^{2}-2 R_{i j i k}\left\langle d \phi e_{j}, d \phi e_{k}\right\rangle\right\} d V_{S M}
$$


Put $d \phi e_{i}=\sum_{\alpha} a_{\alpha i} \bar{e}_{\alpha}$ and $X_{\alpha}=\sum_{i} a_{\alpha i} e_{i}$. If $\operatorname{Ric}^{M}>Q$, then we have

$$
\sum_{A} I\left(d \phi V_{A}, d \phi V_{A}\right) \leq \frac{1}{C_{n-1}} \int_{S M}(n-2 Q)|d \phi|^{2} d V_{S M} .
$$

From (3.27), we immediately obtain

TheOREM 3.5. Let $M^{n}(n \geq 3)$ be a compact Riemannian minimal submanifold of a Riemannian unit sphere with flat normal bundle. If $\mathrm{Ric}^{M}>$ $n / 2$, then there is no non-degenerate stable harmonic map from $M$ to any Finsler manifold.

When the target manifold is Riemannian, let $\left\{\bar{e}_{\alpha}\right\}$ and $\left\{e_{i}\right\}$ be the orthonormal frame of $\bar{M}$ and $M$ respectively. The second variation formula for the harmonic map $\phi: \bar{M}^{m} \rightarrow M^{n}$ can be written as

$$
I(V, V)=\frac{1}{C_{n-1}} \int_{S \bar{M}}\left\{\left\langle\widetilde{\nabla}_{\bar{e}_{\alpha}} V, \widetilde{\nabla}_{\bar{e}_{\alpha}} V\right\rangle-\left\langle R\left(d \phi \bar{e}_{\alpha}, V\right) V, d \phi \bar{e}_{\alpha}\right\rangle\right\} d V_{S \bar{M}} .
$$

Let $d \phi \bar{e}_{\alpha}=a_{\alpha i} e_{i}$, by (3.1). We readily get

$$
\sum_{A} I\left(V_{A}, V_{A}\right)
$$

$$
\begin{aligned}
& =\sum_{A} \frac{1}{C_{n-1}} \int_{S \bar{M}}\left\{a_{\alpha i} a_{\alpha j}\left\langle\nabla_{e_{i}} V_{A}, \nabla_{e_{j}^{H}} V_{A}\right\rangle-a_{\alpha i} a_{\alpha j}\left\langle R\left(e_{i}, V_{A}\right) V_{A}, e_{j}\right\rangle\right\} d V_{S \bar{M}} \\
& =\frac{1}{C_{n-1}} \int_{S \bar{M}}\left\{a_{\alpha i} a_{\alpha j} h_{i k}^{\alpha} h_{j k}^{\alpha}+|d \phi|^{2}-a_{\alpha i} a_{\alpha j}\left\langle R\left(e_{i}, e_{h}\right) e_{k}, e_{j}\right\rangle\right\} d V_{S \bar{M}} .
\end{aligned}
$$

Putting $\operatorname{Ric}^{M}>Q$, using the Gauss equation and (3.29), we obtain

$$
\sum_{A} I\left(V_{A}, V_{A}\right) \leq \frac{1}{C_{n-1}} \int_{S \bar{M}}(n-2 Q)|d \phi|^{2} d V_{S \bar{M}}
$$

From (3.30), we deduce

TheOREM 3.6. There is no non-degenerate stable harmonic map from any compact Finsler manifold to a Riemannian minimal submanifold $M^{n}$ $(n \geq 3)$ of a Riemannian unit sphere with $\operatorname{Ric}^{M}>n / 2$.

Combining Theorems 3.1 and 3.2 shows Theorem 1.1.

Because the Riemannian product $S^{m}(1 / 2) \times S^{m}(1 / 2) \times S^{m}(1 / 2) \times S^{m}(1 / 2)$ is a compact minimal submanifold of $S^{4 m+3}(1)$ with $\operatorname{Ric}^{M}=4(m-1)$ and the Clifford torus $S^{m}(\sqrt{1 / 2}) \times S^{m}(\sqrt{1 / 2})$ is a compact minimal hypersurface of $S^{2 m+1}(1)$ with $\operatorname{Ric}^{M}=2(m-1)$, we immediately derive

COROLlaRY 3.7. There is no non-degenerate stable harmonic map between any compact Finsler manifold and a minimal Clifford hypersurface $S^{m}(\sqrt{1 / 2}) \times S^{m}(\sqrt{1 / 2})(m>2)$. 
COROLlary 3.8. There is no non-degenerate stable harmonic map between any compact Finsler manifold and the Riemannian product $S^{m}(1 / 2) \times$ $S^{m}(1 / 2) \times S^{m}(1 / 2) \times S^{m}(1 / 2)(m>2)$.

Acknowledgements. The author wishes to thank the referee for a careful reading of the manuscript and for very helpful suggestions.

This research was partly supported by NNSF of China (10971170).

\section{References}

[BCS] D. Bao, S. S. Chern and Z. Shen, An Introduction to Riemann-Finsler Geometry, Springer, 2000.

[HS] Q. He and Y. B. Shen, Some results on harmonic maps for Finsler manifolds, Int. J. Math. 16 (2005), 1017-1031.

[L] J. T. Li, Stable P-harmonic maps between Finsler manifolds, Pacific J. Math. 137 (2008), 121-135.

[M] X. H. Mo, Harmonic maps from Finsler manifolds, Illinois J. Math. 45 (2001), $1331-1345$.

[SZ] Y. B. Shen and Y. Zhang, The second variation of harmonic maps between Finsler manifolds, Sci. China Ser. A 47 (2004), 39-51.

Jintang Li

Department of Mathematics

Xiamen University

361005 Xiamen, Fujian, China

E-mail: dli66@xmu.edu.cn

Received 30.9.2009

and in final form 8.12.2009

(2089) 
